\title{
Gait analysis in hip viscosupplementation for osteoarthritis: a case report
}

\section{Di Lorenzo}

Rehabilitation Unit, Gait Analysis Lab, Neuroscience Department, "RUMMO" Hospital, Benevento, Italy

\begin{abstract}
SUMMARY
Hip is a site very commonly affected by osteoarthritis and the intra-articular administration of hyaluronic acid in the management of osteoarthritic pain is increasingly used. However, the debate about its usefulness is still ongoing, as not all results of clinical trials confirm its effectiveness. In order to achieve the best outcome, clinical assessment and treatment choices should be based on subjective outcome, pathological and mechanical findings that should be integrated with qualitative analysis of human movement. After viscosupplementation, clinical trials often evaluate as endpoint subjective outcomes (i.e. pain visual analogic scale) and static imaging such as radiographs and magnetic resonance imaging. In our clinical practice we use gait analysis as part of rehabilitation protocol to measure performance, enhancement and changes of several biomechanical factors. Taking advantage of available resources (BTS Bioengineering gait analysis Elite System) we studied a patient's gait after ultrasound guided hip injections for viscosupplementation. He showed an early clinical and biomechanical improvement during walking after a single intra articular injection of hyaluronic acid. Gait analysis parameters obtained suggest that the pre-treatment slower speed may be caused by antalgic walking patterns, the need for pain control and muscle weakness. After hip viscosupplementation, the joint displayed different temporal, kinetic and kinematic parameters associated with improved pain patterns.
\end{abstract}

Key words: Osteoarthritis, hip pain, hyaluronic acid, viscosupplementation, gait analysis.

\section{INTRODUCTION}

$\mathrm{H}^{\mathrm{i}}$ ip is a site very commonly affected by osteoarthritis (OA) and the intra-articular administration of hyaluronic acid (HA) in the management of osteoarthritic pain is increasing (1-3). OA of the hip causes both anatomical and mechanical changes in and around the involved joint $(4,5)$. These problems cause walking failure impairing stance and gait in the majority of cases.

In some patients, muscle weakening and wasting spread proximally to the thigh muscles, exacerbate pelvis stabilization problems during gait causing sudden knee bending and lameness (5). Patients consequently need to reduce weight bearing using canes or orthoses to maintain independent ambulation.

The classic kinematic feature is the patient walking with a longer double support time, tending to avoid extreme positions of the joints (6). Hip OA patients generally walk with shorter steps and increased cadence $(6,7)$. Gait abnormalities in hip OA pa- tients can be determined by the attempt to avoid pain by walking slowly to control the speed of heel strike and toe-off.

After viscosupplementation, clinical trials often evaluate subjective outcomes (i.e. pain visual analogic scale, Lequesne hip index) $(8,9)$ and static imaging such as radiographs and magnetic resonance imaging (MRI). With the use of clinical gait analysis, a dynamic instrument, it is possible to describe walking patterns pre- and post-supplementation and evaluate objective improvements.

In this paper we present a patient with a moderate/severe (Lequesne hip index $=7-8$ ) hip involvement who maintained independent ambulation only thanks to appropriate adaptations during movements. The patient received viscosupplementation and a preand a post-procedures evaluation of walking patterns by means of instrumental gait analysis.

The motion analysis integrates biomechanical principles with other aspects of kinesiology in the qualitative analysis of human

\author{
Corresponding author: \\ Luigi Di Lorenzo \\ Rehabilitation Unit \\ Gait Analysis Lab \\ Neuroscience Department \\ "RUMMO" Hospital \\ Via Degli Angeli, 1 - 82100 Benevento, Italy \\ E-mail: luigidilorenzo2005@libero.it
}


movement and evaluation of injection outcomes.

\section{CASE REPORT}

The case presented here is a 75-years-old patient with a grade 3 left hip OA diagnosed after hip radiographs and MRI and classified as Lequesne hip index=7-8 (10). He complained about walking problems due to chronic groin pain associated with a rectus muscle pain. In the last weeks before our assessment, hip pain had become increasingly severe, being associated with rectus and quadriceps muscles contractures and muscle pain also on the left side due to a probable overbearing; therefore canes were prescribed and used until hip viscosupplementation.

The patient was treated with a single intra articular injection of high molecular weight hyaluronic acid, a sterile non-pyrogenic viscoelastic solution manufactured with hyaluronic acid sodium salt, obtained by bacterial fermentation from a fraction of high molecular weight with a range of 1500-2000 kDa. The injection was performed under ultrasound guidance with an antero-superior approach, to ensure accurate placement (3).

Treatment efficacy was assessed through Lequesne index $(8,9)$, visual analogue scale pain quantification, and NSAID intake at baseline, and after 2, 3 and 4 weeks. Significant improvements were observed for most measures of hip function and pain. HA reduced symptoms associated with hip osteoarthritis and no side effects were observed. Gait analysis was performed the day before the injection and 39 days later (Figure 1). It was performed using the ELITE system (BTS, Milan, Italy) with 6 video cameras for the acquisition of kinematic variables.

Two platforms indeed are routinely used to acquire ground reaction forces and calculate the center of pressure displacement.

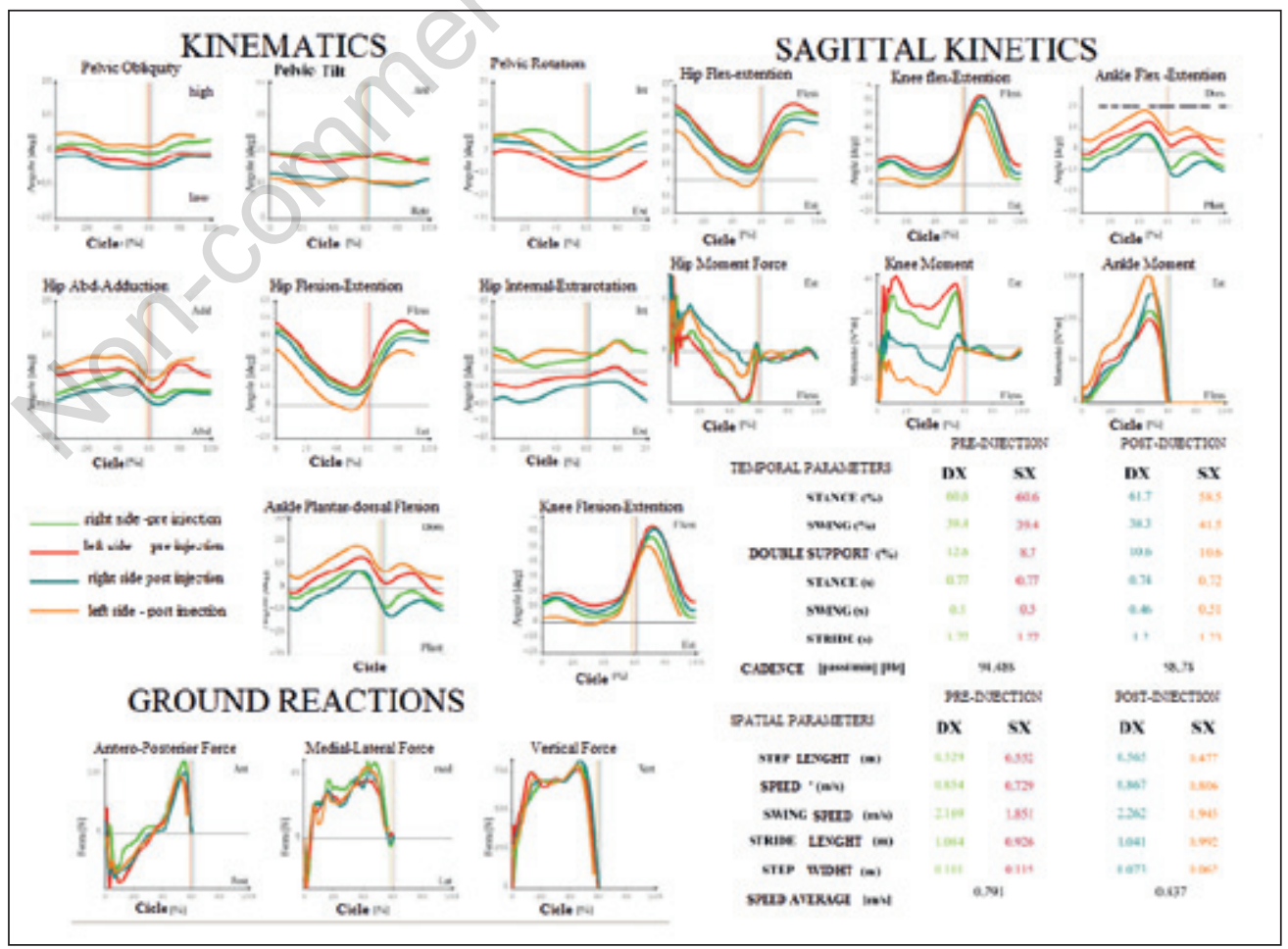

Figure 1 - To evaluate the general characteristics of walking, we considered the following spatio-temporal parameters: right and left stride length, step width $(\mathrm{m})$, duration of the stance phase (expressed as percentage of gait cycle), swing velocity $(\mathrm{m} / \mathrm{s})$, cadence (steps/minute) and mean velocity $(\mathrm{m} / \mathrm{s})$. 
Kinematic and kinetic data were acquired and digitalized. Anthropometric data were collected for the subject and retro reflective spheric markers were placed over prominent bony landmarks. The subject was then instructed to walk at a self-selected speed along a level surface approximately $8 \mathrm{~m}$ in length. All the data were acquired the same day; the mean of 3 valid trials being considered for the analysis. A valid trial was defined as one in which the subject struck the force platforms without adjusting his stride length.

To evaluate the general characteristics of walking, we considered the following spatio-temporal parameters: right and left stride length, step width (m), duration of the stance phase (expressed as percentage of gait cycle), swing velocity $(\mathrm{m} / \mathrm{s})$, cadence (steps/minute) and mean velocity $(\mathrm{m} / \mathrm{s})$. The kinematic evaluation was performed by analyzing the curve profiles of the hip, knee and ankle joint flexion-extension ROM over a gait cycle. Gait analysis results and time-distance parameters were collected and compared together with kinetics data describing the forces that cause the movement.

Kinetic variables are obviously important in gait analysis because they give information on what causes the movement of the joint or the limb, movement strategies and neural compensation.

Data showed in the figure represent the kinetic and kinematic profiles of our patient's hip, knee and ankle joints. The results at baseline show that our patient tended to walk slowly with a longer gait cycle, a shorter step length, a longer double support time and a lower cadence.

The post-procedure increment in walking speed could be related to a decrease of hip joint stiffness thanks to viscosupplementation. Parameters obtained seem to suggest that the pre-treatment slower speed may be caused by antalgic walking patterns, the need for pain control and muscle weakness. With a good pain relief during swing, the hip joint showed, for example, different anterior pelvic tilt and a different and reduced pelvic rotation during walking. Static features were the extreme reduction of painful tendinitis of rectus femoral muscle (diagnosed by ultrasound and clinical examinations) and reduction of trigger points in tight adductor muscle apparently due to the prevalence of the adductor moments at the hip during gait.

\section{DISCUSSION AND CONCLUSIONS}

Viscosupplementation's purpose is to restore the rheological properties, namely viscosity and elasticity, of synovial fluid by normalizing the concentration and molecular weight of hyaluronan $(2,3,11)$. Its benefits are not limited to restoring the mechanical function of the synovial fluid, but further induce the other important roles that hyaluronan plays in the synovial joint (11). Clinical gait analysis offers an objective documentation of the patient's status, can help in treatment planning and assists in the pre/post-treatment comparison.

Alterations in pelvic kinematics have been extensively described in subjects with OA of the hip, in which sagittal plane alterations of pelvic mobility are generally interpreted as compensatory mechanisms to obtain better stride characteristics $(6,12)$. In the last twenty years, kinematics, kinetics and electromyography have profoundly changed the rehabilitative approach. In their prospective trial, Paoloni et al. (12) studied 20 hip OA patients observing the clinical effects of 3 weekly intra-articular injections of $2 \mathrm{~mL}$ of hyaluronic acid in the hip in terms of pain and function at 1 (T1), 3 (T2) and 6-month (T3) follow-ups, as well as of changes in the kinematics and kinetics of gait at 6-month follow-up. They reported that pain significantly dropped after this procedure with a significant improvement of stiffness and disability ( $\mathrm{P}=0.04)$, as measured by the Western Ontario and McMaster Universities OA index.

Gait analysis performed after 6 months, showed that patients at T3 walked with higher cadence and stride length. Moreover, a significant increase for the pelvic tilt at heel contact and for hip flexion-extension moment at loading response sub- 
phases of gait cycle was noted at T3. Pain reduction after HA injection is generally considered to be the result of HA effects on osteoarthritic joint. In fact, our patient experienced a good pain relief few days after a single intra-articular injection of HA, accompanied by kinematic changes in walking pattern after only 39 days suggesting that HA supplementation also provides significant analgesic effects within the joint space.

The patient tend to walk slowly with a longer gait cycle and altered spatio-temporal parameters, confirming that the speed-dependent gait variables are affected by hip pain, caused by structural damage. Viscosupplementation, reducing joint pain and stress, here reduces the tendency to walk slower. Future research should focus on the standardization of measurement methodology for a proper full validation of eventual gait improvements due to hip viscosupplementation.

Conflict of interests: the author declares no potential conflict of interests.

\section{REFERENCES}

1. Arrich J, Piribauer F, Mad P, Schmid D, Klaushofer K, Mullner M. Intra-articular hyaluronic acid for the treatment of the knee: systematic review and meta-analysis. CMAJ. 2005; 172: 1039-43.

2. Foti A, Cisari C, Carda S, Giordan N, Rocco A, Della Bella G. Prospective observational study of the clinical efficacy and safety of intra-articular sodium hyaluronate in synovial joints with osteoarthritis. Eur J Phys Rehabil Med. 2011; 47: 407-15.

3. Migliore A, Tormenta S. Ultrasound guided intra-articular hip injection: technique and clinical application. $1^{\text {St }}$ edition. Roma: Verduci Editore; 2012.

4. Lesher JM, Dreyfuss P, Hager N, Kaplan M, Furman M. Hip joint pain referral patterns: a descriptive study. Pain Med. 2008; 9: 22-5.

5. Di Lorenzo L, Yanow J, Pappagallo M. Psoas impingement syndrome in hip osteoarthritis Joint Bone Spine. 2009; 76: 98-100.

6. Kubota M, Shimada S, Kobayashi S, Sasaki S, Kitade I, et al. Quantitative gait analysis of patients with bilateral hip osteoarthritis excluding the influence of walking speed. J Orthop Sci. 2007; 12: 451-7.

7. Baan H, Dubbeldam R, Nene AV, van de Laar MA. Gait analysis of the lower limb in patients with rheumatoid arthritis: a systematic review. Semin Arthritis Reum. 2012; 41: 768-88.

8. Reips D, Funke F. Interval level measurement with visual analogue scales in Internet-based research: VAS Generator. Behav Res Methods. 2008; 40: 699-704.

9. Lequesne MG. The algofunctional indices for hip and knee osteoarthritis. J Rheumatol. 1997; 24: 779-81.

10. Li KC, Higgs J, Aisen AM, Buckwalter KA, Martel W, McCune WJ. MRI in osteoarthritis of the hip: gradations of severity. Magn Reson Imaging. 1988; 6: 229-36.

11. Carpenter B, Motley T. The role of viscosupplementation in the ankle using Hylan G-F20. J Foot Ankle Surg. 2008; 47: 377-84.

12. Paoloni M, Di Sante L, Dimaggio M, Bernetti A, Mangone M, Di Rienzo S et al. Kinematic and kinetic modifications in walking pattern of hip osteoarthritis patients induced by intraarticular injections of hyaluronic acid. Clin Biomech. 2012; 27: 661-5. 